\title{
A Hall probe diagnostic for low density plasma accelerators
}

\author{
Matthew T. Domonkos, Alec D. Gallimore, ${ }^{\text {a) }}$ and Sven G. Bilén \\ The Plasmadynamics and Electric Propulsion Laboratory, The University of Michigan, Ann Arbor, \\ Michigan 48109
}

(Received 20 October 1997; accepted for publication 4 March 1998)

\begin{abstract}
A Hall probe diagnostic was developed for use with plasma thrusters. The Hall generators were mounted at the end of a stainless steel tube heat exchanger. Ceramic cement and a Pyrex tube were used as radiation and particle flux shields, respectively. A thermocouple was used to monitor the temperature of the Hall generator. The low voltage output from the probe was amplified within one meter of the transducer to minimize the effect of noise pick up. A National Institute of Standards and Technology traceable Gaussmeter provided an absolute reference for calibration, and relative calibrations were performed both in a strong electric field and in situ during thruster operation to approximate the conditions in the discharge. The overall accuracy of the diagnostic was $\pm 6 \mathrm{G}$. The probes were tested with a Hall-effect thruster, and provided sufficiently accurate data to estimate the magnitude of the closed-drift electron current. (C) 1998 American Institute of Physics.
\end{abstract}

[S0034-6748(98)00306-2]

\section{INTRODUCTION}

With electric thrusters entering an era of application on spacecraft, continued improvement in operating efficiency will rely increasingly upon detailed understanding of the physics governing the operation of these devices. Magnetic fields generated by electromagnets or permanent magnets in electric thrusters can be easily measured using an ordinary Gaussmeter or a calibrated Hall generator. However in the case of Hall-effect and magnetoplasmadynamic thrusters, large currents in the plasma are thought to contribute significantly to the overall magnetic field topography. ${ }^{1}$ With these types of thrusters, both ac and dc magnetic field information within the discharge is required to validate theories of thruster operation. This article describes the design and application of Hall probes to measure the magnetic field in an operating plasma accelerator.

Measurement of the steady magnetic field generated in the Hall-effect anode layer thruster (TAL) was the primary goal of this investigation, although the probe design can be generalized to other applications. The TAL used in this investigation has an annular discharge chamber with a mean diameter of $55 \mathrm{~mm}$, and this constrained the probe dimensions both to minimize the perturbation to the plasma and to provide spatial resolution to about $1 \mathrm{~mm}$. A Hall generator was chosen as the transducer due to the relative ease of measuring the steady component of the magnetic field compared to a magnetic coil. ${ }^{2,3}$ Since the Hall generator is a semiconductor device and must operate below $100{ }^{\circ} \mathrm{C}$, the plasma energy deposition was evaluated to provide a basis for the thermal protection of the probe. Hall-effect thrusters have electron number densities and temperatures as high as 8 $\times 10^{12} \mathrm{~cm}^{-3}$ and $10 \mathrm{eV}$, respectively. ${ }^{4,5}$ A simple experiment showed that the particle and radiative fluxes upon a $12.8 \mathrm{~mm}$ diam rod left in the near-field plume of the TAL were suffi-

${ }^{a}$ Electronic mail: alec.gallimore@umich.edu cient to heat it to greater than $200{ }^{\circ} \mathrm{C}$. Since the Hall sensor was limited to operation below $100^{\circ} \mathrm{C}$, active water cooling was employed in addition to radiative and particle flux shields. The magnetic field in Hall thrusters typically peaks at several hundred Gauss, and for these tests, resolution to several Gauss was deemed necessary to resolve the topography. The need for resolution to within several Gauss drove the design of the thermal protection, support structure, signal conditioning, and diagnostic procedures.

This article begins by detailing the design of the Hall probes developed to meet the test constraints. Next, the probe calibration procedure is examined with emphasis placed on the calibration conditions. The overall experimental method is then presented, followed by some results obtained with the probes.

\section{PROBE DESIGN}

Figure 1(a) shows a mechanical schematic of the transverse Hall probe which is a derivative of the design used by Myers. ${ }^{1}$ The transducers were F. W. Bell BH-209 (transverse) and BH-208 (axial) Hall generators. The generator dimensions are summarized in Table I. The size was chosen primarily to minimize the overall dimensions of the probe assembly, thereby reducing the perturbation to the plasma. The generators were mounted to nonmagnetic stainless steel cooling tubes using ceramic cement. The ceramic acted as a conduction path between the cooling lines and the generator, and as a radiation shield between the sensor and the plasma. A type $\mathrm{K}$ thermocouple made with 36 AWG wire was mounted next to the Hall generator so that its operating temperature could be monitored. Cooling water was pumped through the stainless steel tubes with a VWR Scientific refrigerated recirculator which maintained an outlet temperature of $7{ }^{\circ} \mathrm{C}$. A single-ended Pyrex tube protected the probe assembly from the particle flux of the plasma. Glass fiber sleeves insulated the stainless steel tubes from the plasma. 
(a)

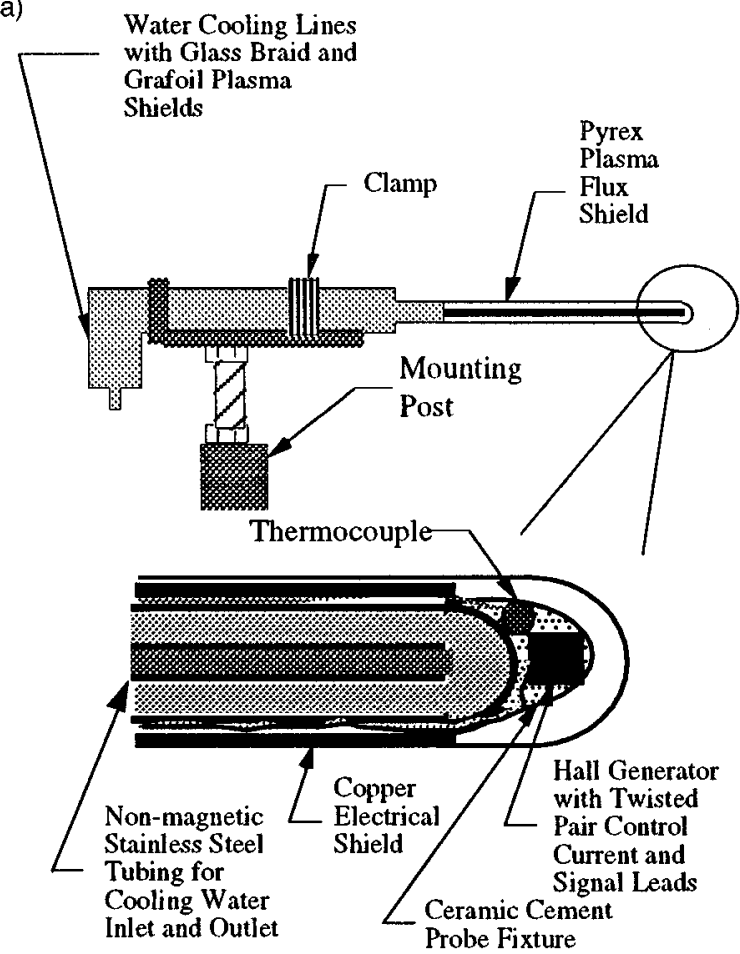

(b)

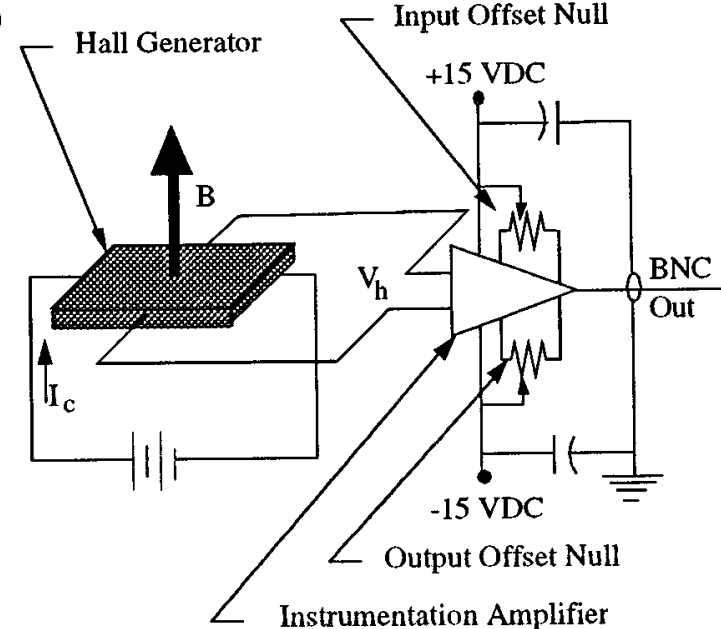

FIG. 1. Schematic of the water-cooled Hall probes used in the D55 tests. (a) Mechanical diagram, (b) electrical schematic.

The electrical design is illustrated in Fig. 1(b). Given the stated sensitivity of these probes and the goal of resolution within several Gauss, accurate measurement of the probe output to approximately $10 \mu \mathrm{V}$ was necessary. To achieve this, a 1000 gain Analog Devices 524AD precision instrumentation amplifier was used. It was placed in the vacuum chamber within one meter of the generator to amplify the cleanest possible signal while isolating its semiconductor elements from the plasma. This signal was then sent through shielded-twisted-pair and coaxial cables to a digital oscillo-

TABLE I. Dimensions of the Hall generators.

\begin{tabular}{ccc}
\hline \hline Sensor & Length $(\mathrm{mm})$ & Width $(\mathrm{mm})$ \\
\hline BH 208 (axial) & 1.02 & 0.51 \\
BH 209 (trans) & 1.02 & 0.51 \\
\hline \hline
\end{tabular}

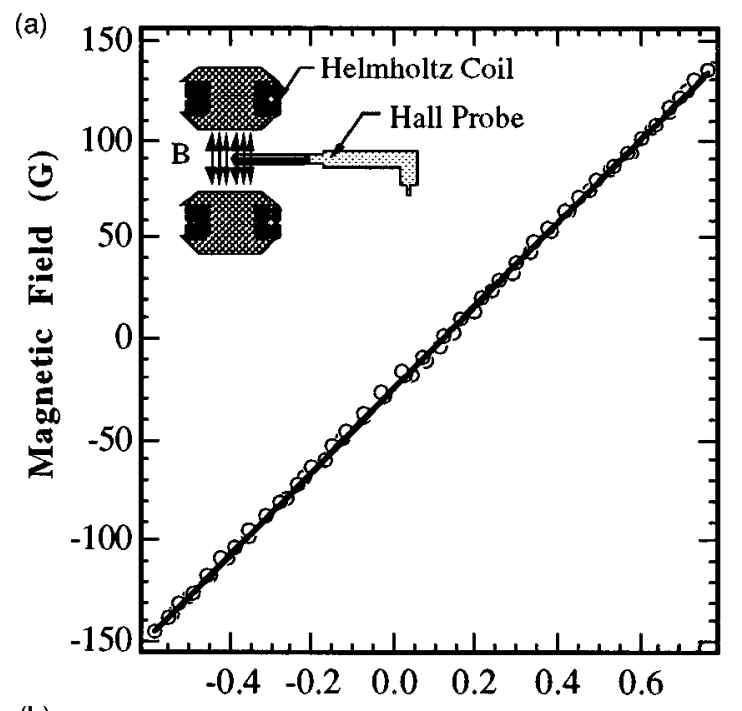

(b)

Hall Probe Voltage (V)

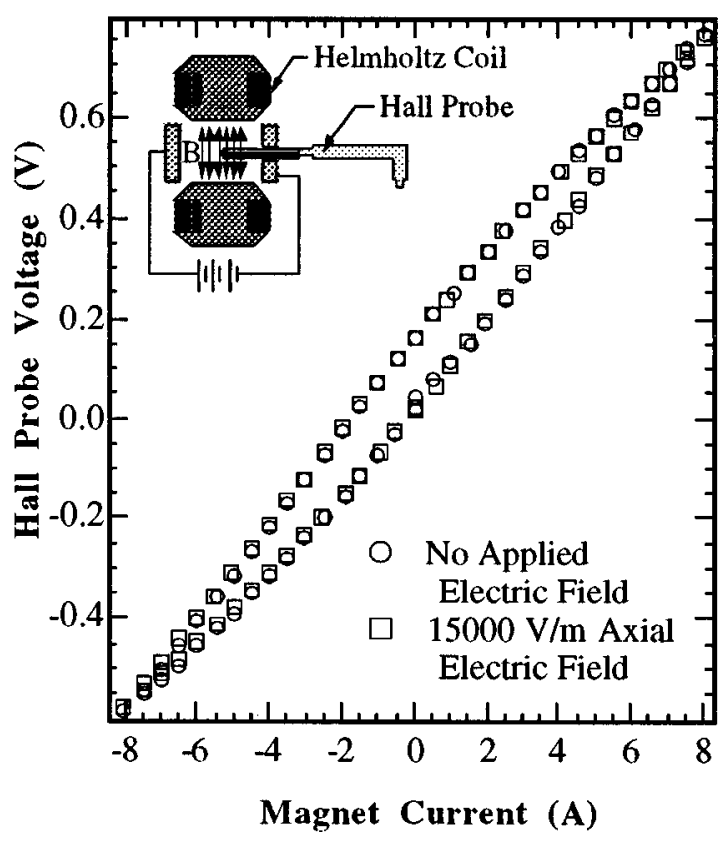

FIG. 2. Calibration of the Hall probe. (a) Hall probe calibration curve, (b) evaluation of the effect of a strong electric field on Hall probe response.

scope where data were stored prior to transfer to a computer. The constant probe control current $I_{c}$ was supplied by a Lambda LPD-422A-PM power supply.

\section{PROBE CALIBRATION}

Two different probe calibrations were performed with steady magnetic fields in atmosphere. The first calibration, shown in Fig. 2(a), provided an absolute calibration of the probe with respect to a NIST traceable Walker Scientific MG-5DAR Gaussmeter. The Gaussmeter was accurate to $\pm 1 \mathrm{G}$. The Helmholtz coil provided a magnetic field uniform to within $\pm 1 \mathrm{G}$ over the positions of the Gaussmeter transducer and the Hall probe. This curve shows that the response of the Hall probe is linear and agrees with the Gaussmeter to within \pm 2 G. Table II summarizes the accuracy of the various calibrations and techniques used in the diagnostic. 
TABLE II. Breakdown of the Hall probe diagnostic accuracy.

\begin{tabular}{cc}
\hline \hline Component or effect & Accuracy $(\mathrm{G})$ \\
\hline Gaussmeter & \pm 1 \\
Helmholtz coil & \pm 1 \\
Absolute calibration & \pm 2 \\
Vibration & \pm 1.5 \\
Total & $\sim \pm 6$ \\
\hline \hline
\end{tabular}

Since the probe was intended for use within a few centimeters of the TAL, it would be exposed to an axial electric field on the order of $10^{4} \mathrm{~V} / \mathrm{m}^{6}{ }^{6}$ Consequently, as Fig. 2(b) shows, the probe was also calibrated in the Helmholtz coil with an applied electric field similar in magnitude and orientation to the maximum expected in the thruster ${ }^{6}$ to assess any effects caused by the electric field. As can be seen, the electric field has a negligible effect, if any, on the Hall probe response. The hysteresis loop is indicative of the residual magnetism induced in the Helmholtz coil cores by the applied current. The current was measured using a digital multimeter.

\section{DIAGNOSTIC DESIGN}

The Hall probes were mounted to a two-axis positioning system that would sweep the probe through the plume of the thruster. Magnetic field data were taken continuously during each sweep with the oscilloscope. The sweeps also served to minimize the heat deposition to the probe to maintain a low temperature. If the Hall probe temperature drifted above $24{ }^{\circ} \mathrm{C}$, the probe was left out of the plume for an extended period. By tolerating only small temperature changes, the error due to thermal drift was considerably less than $1 \%$. Vibration in the probe support structure added an uncertainty of approximately $\pm 1.5 \mathrm{G}$, based on the spread in data taken on the magnetic field generated by the thruster electromagnets without a discharge.

A relative in situ calibration was also used to address the effects of the discharge electrical noise and the presence of the plasma on probe response. An electromagnet was placed approximately $0.5 \mathrm{~m}$ from the thruster for calibration purposes. Figure 3 shows a typical comparison between the response in vacuum and during the discharge. Besides the clear change in the offset, the slope is reduced by approximately $2 \%$ during the discharge. Since the effect of the discharge noise can be calibrated out of the measurement, the typical overall accuracy of the probes is $\pm 6 \mathrm{G}$.

\section{TESTS WITH THE ANODE LAYER THRUSTER}

The Hall probes were used to examine the magnetic field of the TAL, shown schematically in Fig. 4, during operation. The TAL was built by the Central Scientific Research Institute for Machine Building of Kaliningrad, Russia, and uses three outer and one inner electromagnets to generate a radial magnetic field to trap electrons in a closed azimuthal drift. The cloud of trapped electrons ionizes neutral xenon injected through the rear of the annular anode, and accelerates ionized xenon axially. The large ion Larmor radius prevents the ions from being trapped by the magnetic field, and the hollow

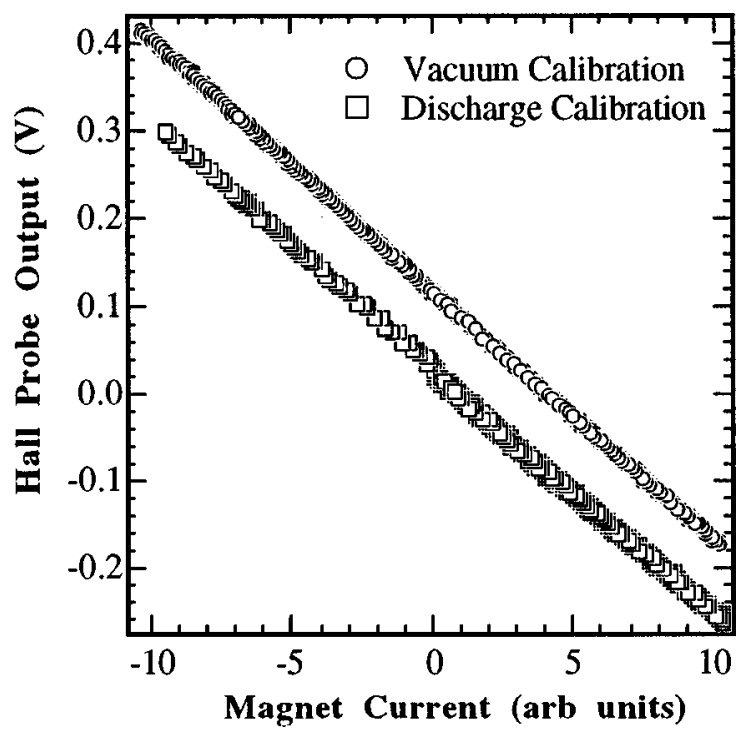

FIG. 3. Comparison of the in situ relative calibrations.

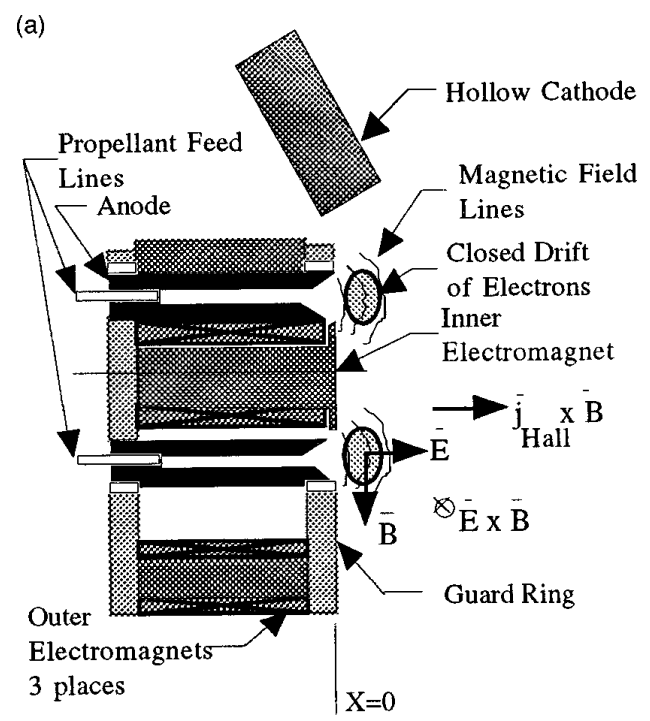

(b)

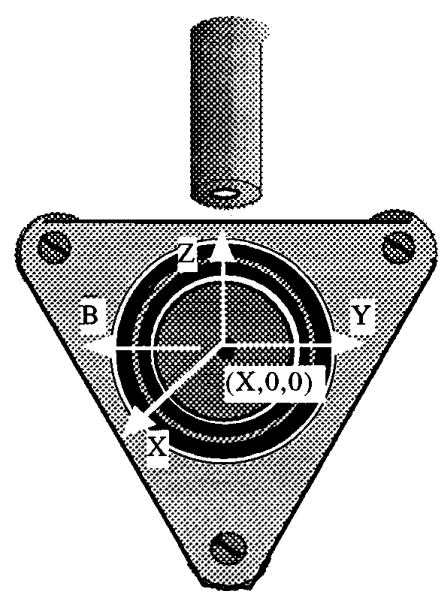

FIG. 4. Schematic of the D55 anode layer thruster. (a) Side section view, (b) front view. 


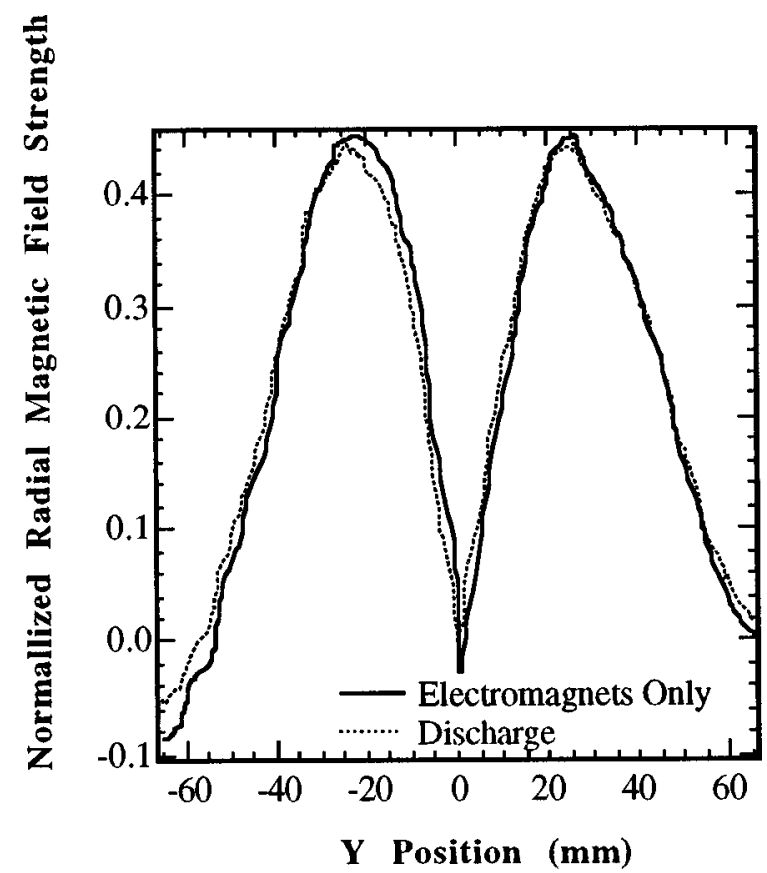

FIG. 5. Normalized comparison of the vacuum field generated by the electromagnets and the field generated during thruster operation. $X=20 \mathrm{~mm}$.

cathode/neutralizer emits electrons to maintain quasineutrality in the accelerated ion beam. Detailed descriptions of the plasma generated by this device and its performance can be found elsewhere. ${ }^{4,7-9}$

The primary goal of the magnetic field investigation was to establish bounds for the magnitude of the closed drift current. The azimuthal drift acts to decrease the overall radial magnetic field, and this effect will become evident when comparing the field generated by the electromagnets alone with the discharge magnetic field. Figure 5 depicts this comparison. Due to the proprietary nature of the magnetic field data, the values reported in this article have been normalized to the maximum vacuum radial field strength $5 \mathrm{~mm}$ from the exit plane, $X=5 \mathrm{~mm}$. The change in field strength is less than 5\%, which limits the azimuthal electron drift current to tens of Amps. This also implies that the magnetic field generated by the electromagnets provides a good approximation of the total field in the discharge of a Hall thruster.

In order to estimate the drift current more accurately, the dimensions and current density distribution of the electron cloud would be required. A crude determination of the extent of the closed drift region was made during attempts to probe within $10 \mathrm{~mm}$ of the exit plane of the thruster. The insulating Pyrex tip of the probe extends approximately $5 \mathrm{~mm}$ beyond the location of the Hall generator, and when the tip of the probe was within $5 \mathrm{~mm}$ of the thruster, the discharge current nearly doubled as the probe swept in front of the anode. Consequently, the closed drift region is believed to extend at least $5 \mathrm{~mm}$ from the exit plane of the thruster. This event also represents one of the limitations of this technique, that the probes can only be used where they leave the plasma relatively undisturbed.

\section{ACKNOWLEDGMENTS}

This work was supported by a research grant from the NumerEx Corporation in conjunction with the Jet Propulsion Laboratory. Dr. Michael Frese of NumerEx and Dr. Keith Goodfellow of JPL monitored the grant.

${ }^{1}$ R. M. Myers, AIAA Paper No. 89-2832, July 1989.

${ }^{2}$ H. Tahara, M. Sasaki, Y. Kagaya, and T. Yoshikawa, AIAA Paper 902554, July 1990.

${ }^{3}$ P. Tschang, AIAA J. 3, 849 (1965).

${ }^{4}$ M. T. Domonkos, C. M. Marrese, J. H. Haas, and A. D. Gallimore, AIAA Paper No. 97-3062, July 1997.

${ }^{5}$ S. W. Kim, J. E. Foster, and A. D. Gallimore, AIAA Paper 96-2972, July 1996.

${ }^{6}$ A. M. Bishaev and V. Kim, Sov. Phys. Tech. Phys. 23, 1055 (1978).

${ }^{7}$ C. E. Garner, J. R. Brophy, J. E. Polk, S. Semenkin, V. Garkusha, S. Tverdokhlebov, and C. M. Marrese, "Experimental Evaluation of the Russian Anode Layer Thruster," Third Russian-German Conference on Electric Propulsion Engines and Their Technical Applications, Stuttgart, Germany, 19-23 July 1994 (unpublished).

${ }^{8}$ C. M. Marrese, J. E. Polk, L. B. King, A. D. Gallimore, S. Semenkin, V. Garkusha, and S. Tverdokhlebov, 24th International Electric Propulsion Conference, Moscow, Russia, 1995 (unpublished), Vol. 2, pp. 1326-1333, IEPC Paper No. 95-196.

${ }^{9}$ A. I. Bugrova, A. I. Morozov, and V. K. Kharchevnikov, Sov. Phys. Tech. Phys. 30, 610 (1985). 